\title{
The Correlation between Maternal Age and Fetal Sex Chromosome Aneuploidies: A 8-year Single Institution Experience in China
}

\author{
Li Hongge ( $\square$ fbyc2@zju.edu.cn ) \\ Zhejiang University School of Medicine Women's Hospital \\ Jinglei Jin \\ Zhejiang University School of Medicine Women's Hospital
}

\section{Research}

Keywords: Maternal age, Sex chromosome aneuploidies (SCAs), Second-trimester amniocentesis, Prenatal diagnosis, Cytogenetic diagnosis

Posted Date: December 16th, 2020

DOI: https://doi.org/10.21203/rs.3.rs-127027/v1

License: (c) (i) This work is licensed under a Creative Commons Attribution 4.0 International License. Read Full License 


\section{Abstract}

Background: Although a few studies have investigated the association between maternal age and fetal sex chromosome aneuploidies (SCAs), most of these studies were limited to advanced age women and the results were conflicting. This study aimed to investigate maternal agespecific rates for common fetal SCAs (including $45, X, 47, X X Y, 47, X X X$ and $47, X Y Y$ ) in pregnant women of different ages that not only limited to advanced age women. We retrospectively investigated a 8-year experience of prenatal diagnosis for fetal chromosome aberrations by secondtrimester amniocentesis at a university teaching hospital in China. 20,409 amniotic fluid specimens collected at 19-22 ${ }^{+6}$ gestational weeks were included in this study. The women were categorized into five age groups $\left(\leq 23,24-28,29-33,34-38,39^{+}\right.$years $)$based on maternal age at the time of amniocentesis and entered as a categorical variable in all samples. The correlation between fetal SCAs and maternal age was determined using the logistic regression analysis. A chi-square test was performed to compare the incidence of fetal SCAs among age groups.

Results: A total of 179 cases of fetal SCAs were detected, and the incidence was 8.77\%o (about 1/114). The incidence of all SCAs increased significantly with advancing maternal age (SE, 0.014; odds ratio, 1.044; $P=0.002$ ), from 5.81 per 1,000 fetuses at the $24-28$ years to 10.92 per 1,000 at the $39+$ years. The incidence of all SCAs was also significantly different among age groups $\left(X^{2}=10.197, P=0.037<0.05\right)$. Specifically, the incidence of $45, \mathrm{X}(\mathrm{SE}, 0.037$; odds ratio, 0.916 ; $P=0.017)$ and $47, \mathrm{XXY}(\mathrm{SE}, 0.024$; odds ratio, 1.127 ; $P=0.000)$ had significant correlation with maternal age, while the incidence of $47, \mathrm{XXX}$ and $47, \mathrm{XYY}$ had no correlation with maternal age $(P=0.473 ; P=0.272$, respectively).

Conclusions: Maternal age was ascertained to be a strong risk factor for fetal SCAs.

\section{Backgroud}

Chromosomal abnormalities are the leading causes of stillbirth and neonatal birth defects [1]. The most common numerical chromosomal abnormalities observed in the liveborns are Down syndrome (trisomy 21 or T21), Edward syndrome (trisomy 18 or T18), Patau syndrome (trisomy 13 or T13), and sex chromosome aneuploidies (SCAs) [2]. SCAs occur with a frequency of 1 in 500, an incidence greater than that of trisomy 21 [3]. SCAs are a common group of chromosome disorders characterized by the loss or gain of one or more sex chromosomes, including 45,X (Turner syndrome; 1/2000 female livebirths), 47,XXX (Triple X syndrome; 1/1000 female livebirths), 47,XXY (Klinefelter syndrome; $1 / 600$ male livebirths), 47,XYY (47,XYY syndrome; $1 / 1000$ male livebirths), as well as rare SCAs such as $48, X X X X, 48, X X X Y, 48, X X Y Y$ and $69, X X X$ [4-5]. The karyotype analysis of amniotic fluid cells in the second-trimester is the main method for detecting fetal chromosomal aberrations and is regarded as the gold standard for cytogenetic diagnosis currently [6].

A number of risk factors can increase the probability of chromosomal abnormalities during pregnancy. Since the 1980 s, it is clearly shown that maternal age could be the most important risk factor for fetal chromosomal abnormalities [7]. To date, it has been widely accepted that the risk of autosomal aneuploidies (including T21, T18 and T13) is directly related to the increase in maternal age [8]. Unfortunately, although a few studies have investigated the association between maternal age and fetal SCAs including 45, X, 47,XXY, 47,XXX and 47,XYY, most of these studies were limited to advanced age women and the results were conflicting. In 1984, Ferguson-Smith et al [9] analyzed maternal age-specific rates for chromosomal aberrations in 52,965 pregnancies aged 35 years and older from Europe and outside Europe, and the collaborative study confirmed that 47,XXX and 47,XXY increased with advancing maternal age, while 47,XYY did not. In addition, a significant inverse relationship with maternal age was found for 45,X. In 2015, Zhu Y et al [10] reviewed 46,258 advanced age women who received amniocentesis and karyotype analysis during the second-trimester from 13 prenatal diagnosis centers in China, and concluded that 47,XXX and 47,XXY increased with advancing maternal age, while 45,X and 47,XYY were not significantly associated with maternal age. In 2009, Forabosco A et al [11] collected the results of 88,965 amniocentesis (including 51,758 cases with maternal age $\geq 35$ years and 37,207 cases with maternal age < 35 years) from all cytogenetics laboratories in Italy and found that 47,XXX and 47,XXY were largely age-related with maternal age, while 47,XYY and 45,X showed a borderline significance. In 2013, Kim et al [12] targeted 15,381 advanced age women who underwent amniocentesis in South Korea and found that no significant correlation with maternal age was found for $45, X, 47, X X X$, and $47, X X Y$. Since $47, X Y Y$ was found in only two subjects, analysis of the correlation between 47,XYY and maternal age was not possible. In summary, several large-scale epidemiological studies on the relationship between maternal age and chromosomal abnormalities were multicenter collaborative studies, and almost all of these studies were limited to advanced age women and these conclusions were conflicting.

The intention of this study was to investigate the correlation between maternal age and common fetal SCAs (including $45, X, 47, X X Y, 47, X X X$ and $47, X Y Y)$. Here, we retrospectively investigated a single center experience of prenatal diagnosis for fetal chromosomal aberrations during the last 8 years (from January 1st 2011, until December 31st 2018) in the Women's Hospital of Zhejiang University, a university teaching hospital, in southern China. Unlike previous publications, this study compared the incidence of fetal SCAs in pregnant women of different ages that were not limited to women of advanced ages, and this study did not exclude pregnant women with family history of chromosomal abnormalities and fetal abnormalities detected by ultrasound or other indications of an unfavorable prenatal diagnosis, which could better reflect the maternal agerelated risk of fetal SCAs. 
Results

\section{Clinical characteristics of the entire cohort in the study.}

This study included 20,409 singleton pregnancies and 186 cases of fetal SCAs were diagnosed. The clinical significance of fetal SCAs (by year) in the entire cohort is shown in Table 1. Excluding 7 rare fetal SCAs (including 1 case of 48,XXXY, 2 cases of 48,XXXX, 1 case of 48,XXYY and 3 cases of $69, X X X), 179$ cases of common fetal SCAs were diagnosed with an incidence rate of $8.77 \%$ (about 1/114). The most common fetal SCAs was $47, X X Y$ in 70 cases (39.11\%), followed by $47, X X X$ in 44 (24.58\%), 47,XYY in 39 (21.79\%) and 45,X in 26 (14.53\%). The proportion of advanced age women receiving amniocentesis accounted for about half $(45.71 \%, 9329 / 20409)$ of the clinical samples. Change in the relative number of amniocentesis and advanced age women $(\geq 35)$ undergoing amniocentesis by year are showed in Fig. 1.(a). A trend in the proportion of women with advanced maternal age undergoing amniocentesis and mean age of the women are compared in Fig. 1.(b). The results showed that the proportion of AMA women and the mean age of the women have been increasing since 2012, and especially in 2016 and 2017 , then reduced in 2018. Age of these women ranged from 18 to 52 years, with an average age of 33.09 years $( \pm 5.447)$. Maternal age distribution of the entire study cohort and the incidence (per 1000) of fetal SCAs by maternal age are shown in Fig. 2 and Table 2. Two birth peaks appeared at 28 and 35 years old. The incidence of $47, X X Y, 47, X X X$ and $47, X Y Y$ was found to increase dramatically at the age of 44 .

Table 1

Clinical significance of fetal SCAs (by year) in the entire cohort.

\begin{tabular}{|c|c|c|c|c|c|c|c|c|c|c|c|c|c|}
\hline \multirow[t]{2}{*}{ Year } & \multirow{2}{*}{$\begin{array}{l}\text { No. of } \\
\text { patients }\end{array}$} & \multirow[t]{2}{*}{ AMA } & \multirow{2}{*}{$\begin{array}{l}\% \text { of } \\
\text { AMA }\end{array}$} & \multirow{2}{*}{$\begin{array}{l}\text { Mean } \\
\text { age } \pm \\
\text { SD }\end{array}$} & \multicolumn{9}{|c|}{ Sex chromosome aneuploidies } \\
\hline & & & & & Total & $45, X$ & $47, X X Y$ & $47, X X X$ & $47, X Y Y$ & $48, X X X Y$ & $48, X X X X$ & $48, X X Y Y$ & $69, X X X$ \\
\hline 2011 & 3314 & 1518 & $45.81 \%$ & $\begin{array}{l}32.74 \\
\pm \\
5.359\end{array}$ & 9 & 2 & 1 & 2 & 2 & 1 & & & 1 \\
\hline 2012 & 2364 & 895 & $37.86 \%$ & $\begin{array}{l}32.03 \\
\pm \\
5.430\end{array}$ & 10 & 3 & 3 & 1 & 2 & & & & 1 \\
\hline 2013 & 1840 & 697 & $37.88 \%$ & $\begin{array}{l}32.20 \\
\pm \\
5.476\end{array}$ & 16 & 4 & 7 & 2 & 2 & & 1 & & \\
\hline 2014 & 2520 & 1026 & $40.71 \%$ & $\begin{array}{l}32.47 \\
\pm \\
5.282\end{array}$ & 13 & 4 & 2 & 4 & 3 & & & & \\
\hline 2015 & 2097 & 955 & $45.54 \%$ & $\begin{array}{l}32.85 \\
\pm \\
5.347\end{array}$ & 14 & 1 & 6 & 3 & 4 & & & & \\
\hline 2016 & 2309 & 1294 & $56.04 \%$ & $\begin{array}{l}34.26 \\
\pm \\
5.143\end{array}$ & 32 & 3 & 13 & 8 & 7 & & & 1 & \\
\hline 2017 & 2154 & 1240 & $57.57 \%$ & $\begin{array}{l}34.49 \\
\pm \\
5.456\end{array}$ & 22 & 1 & 10 & 5 & 4 & & 1 & & 1 \\
\hline 2018 & 3811 & 1704 & $44.71 \%$ & $\begin{array}{l}33.54 \\
\pm \\
5.530\end{array}$ & 70 & 8 & 28 & 19 & 15 & & & & \\
\hline Total & 20409 & 9329 & $45.71 \%$ & $\begin{array}{l}33.09 \\
\pm \\
5.447\end{array}$ & 186 & 26 & 70 & 44 & 39 & 1 & 2 & 1 & 3 \\
\hline
\end{tabular}


Table 2

The incidence (per 1000) of 45,X,47,XXY, 47,XXX and 47,XYY by maternal age in the study.

\begin{tabular}{|c|c|c|c|c|c|c|}
\hline Maternal age & No. of patients & $45, x$ & $47, X X Y$ & $47, X X X$ & $47, X Y Y$ & All SCAs \\
\hline 18 & 7 & $0(0)$ & $0(0)$ & $0(0)$ & $0(0)$ & $0(0)$ \\
\hline 19 & 15 & $0(0)$ & $0(0)$ & $0(0)$ & $0(0)$ & $0(0)$ \\
\hline 20 & 34 & $0(0)$ & $0(0)$ & $0(0)$ & $0(0)$ & $0(0)$ \\
\hline 21 & 100 & $0(0)$ & $0(0)$ & $0(0)$ & $0(0)$ & $0(0)$ \\
\hline 22 & 188 & $0(0)$ & $1(5.32)$ & $1(5.32)$ & $0(0)$ & $2(10.64)$ \\
\hline 23 & 314 & $1(3.18)$ & $0(0)$ & $1(3.18)$ & $0(0)$ & $2(6.37)$ \\
\hline 24 & 526 & $0(0)$ & $1(1.90)$ & $1(1.90)$ & $0(0)$ & $2(3.80)$ \\
\hline 25 & 666 & $0(0)$ & $1(1.50)$ & $2(3.00)$ & $1(1.50)$ & $4(6.01)$ \\
\hline 26 & 877 & $2(2.28)$ & $0(0)$ & $2(2.28)$ & $2(2.28)$ & $6(6.84)$ \\
\hline 27 & 990 & $5(5.05)$ & $1(1.01)$ & $0(0)$ & $1(1.01)$ & $7(7.07)$ \\
\hline 28 & 1245 & $1(0.80)$ & $1(0.80)$ & $3(2.41)$ & $1(0.80)$ & $6(4.82)$ \\
\hline 29 & 1153 & $5(4.34)$ & $2(1.73)$ & $2(1.73)$ & $1(0.87)$ & 10 (8.67) \\
\hline 30 & 1089 & $2(1.84)$ & $1(0.92)$ & $3(2.75)$ & $0(0)$ & $6(5.51)$ \\
\hline 31 & 1012 & $0(0)$ & $2(1.98)$ & $2(1.98)$ & $3(2.96)$ & $7(6.92)$ \\
\hline 32 & 1002 & $4(3.99)$ & $1(1.00)$ & $1(1.00)$ & $6(5.99)$ & $12(11.98)$ \\
\hline 33 & 841 & $1(1.19)$ & $2(2.38)$ & $0(0)$ & $1(1.19)$ & $4(4.76)$ \\
\hline 34 & 925 & $1(1.08)$ & $4(4.32)$ & $2(2.16)$ & $5(5.41)$ & 12 (12.97) \\
\hline 35 & 1616 & $1(0.62)$ & $7(4.33)$ & $7(4.33)$ & $4(2.48)$ & 19 (11.76) \\
\hline 36 & 1562 & $0(0)$ & $11(7.04)$ & $3(1.92)$ & $2(1.28)$ & $16(10.24)$ \\
\hline 37 & 1421 & $0(0)$ & $6(4.22)$ & $2(2.11)$ & $4(4.22)$ & $12(8.44)$ \\
\hline 38 & 1256 & $1(0.80)$ & $5(3.98)$ & $4(3.18)$ & $3(2.39)$ & 13 (10.35) \\
\hline 39 & 1088 & $1(0.92)$ & $7(6.43)$ & $0(0)$ & $1(0.92)$ & $9(8.27)$ \\
\hline 40 & 866 & $0(0)$ & $5(5.77)$ & $2(2.31)$ & $2(2.31)$ & 9 (10.39) \\
\hline 41 & 644 & $0(0)$ & $5(7.76)$ & $1(1.55)$ & $0(0)$ & $6(9.32)$ \\
\hline 42 & 461 & $0(0)$ & $3(6.51)$ & $1(2.17)$ & $1(2.17)$ & $5(10.85)$ \\
\hline 43 & 241 & $1(4.15)$ & $1(4.15)$ & $1(4.15)$ & $0(0)$ & $3(12.45)$ \\
\hline 44 & 136 & $0(0)$ & $2(14.71)$ & $3(22.06)$ & $1(7.35)$ & $6(44.12)$ \\
\hline 45 & 75 & $0(0)$ & $0(0)$ & $0(0)$ & $0(0)$ & $0(0)$ \\
\hline 46 & 28 & $0(0)$ & $1(35.71)$ & $0(0)$ & $0(0)$ & $1(35.71)$ \\
\hline 47 & 18 & $0(0)$ & $0(0)$ & $0(0)$ & $0(0)$ & $0(0)$ \\
\hline 48 & 8 & $0(0)$ & $0(0)$ & $0(0)$ & $0(0)$ & $0(0)$ \\
\hline 49 & 3 & $0(0)$ & $0(0)$ & $0(0)$ & $0(0)$ & $0(0)$ \\
\hline 51 & 1 & $0(0)$ & $0(0)$ & $0(0)$ & $0(0)$ & $0(0)$ \\
\hline 52 & 1 & $0(0)$ & $0(0)$ & $0(0)$ & $0(0)$ & $0(0)$ \\
\hline Total & 20409 & $26(1.27)$ & 70 (3.42) & $44(2.16)$ & 39 (1.91) & 179 (8.77) \\
\hline
\end{tabular}


In the study, AMA and positive results of MSS were the most frequent reasons for referral, accounting for $47.69 \%$ and $34.65 \%$, respectively. The most of SCAs were detected with the indication of AMA (111/179, 62.01\%), followed by abnormal NIPT results (34/179, 19.00\%), abnormal ultrasound findings $(16 / 179,8.94 \%)$, positive result of MSS $(15 / 179,8.38 \%)$ and others $(3 / 179,1.68 \%)$. However, the incidence of SCAs varied by indications: $83.74 \%$ o for abnormal NIPT results, $17.98 \%$ o for abnormal ultrasound findings, $11.40 \%$ o for AMA, $2.12 \%$ o for positive results of MSS and $1.30 \%$ o for others. AMA was the most frequent referral indication for $47, X X Y, 47, X X X$ and $47, X Y Y$, while abnormal ultrasound findings were the most frequent referral indication for $45, X$. Three pregnant women with the indication of others were found to have fetal SCAs, including 1 case of monogenetic disease, 1 case of a previous child with $5 \mathrm{p}$-, and 1 case of a previous child with 21 trisomy. None of the pregnant women with fetal SCAs were found to have the referral indication of paternal/maternal carrying sex chromosome abnormalities or a family history of a previous child with sex chromosome abnormalities. The incidence and proportion of fetal SCAs by referral indications are showed in Table 3.

Table 3

The frequency and proportion of fetal SCAs by referral indications.

\begin{tabular}{|c|c|c|c|c|c|c|c|}
\hline \multirow[t]{2}{*}{ Referral indications } & \multirow{2}{*}{$\begin{array}{l}\text { No.of patients } \\
(\%)\end{array}$} & \multirow[t]{2}{*}{ SCAs (\%) } & \multirow{2}{*}{$\begin{array}{l}\text { SCAs (\%o) by } \\
\text { indications }\end{array}$} & \multicolumn{4}{|l|}{ SCAs } \\
\hline & & & & $45, X$ & $47, X X Y$ & $47, X X X$ & $47, X Y Y$ \\
\hline AMA & $9734(47.69 \%)$ & $111(62.01 \%)$ & $11.40 \%$ o & $5(19.23 \%)$ & $57(81.43 \%)$ & $26(59.09 \%)$ & $23(58.97 \%)$ \\
\hline $\begin{array}{l}\text { Positive results of } \\
\text { MSS }\end{array}$ & $7072(34.65 \%)$ & $15(8.38 \%)$ & $2.12 \%$ & $5(19.23 \%)$ & $1(1.43 \%)$ & $3(6.82 \%)$ & $6(15.38 \%)$ \\
\hline $\begin{array}{l}\text { Abnormal ultrasound } \\
\text { findings }\end{array}$ & $890(4.36 \%)$ & $16(8.94 \%)$ & $17.98 \%$ о & $10(38.46 \%)$ & $2(2.86 \%)$ & $3(6.82 \%)$ & $1(2.56 \%)$ \\
\hline $\begin{array}{l}\text { Abnormal NIPT } \\
\text { results }\end{array}$ & $406(1.99 \%)$ & $34(19.00 \%)$ & $83.74 \%$ o & $6(23.08 \%)$ & $9(12.86 \%)$ & $12(27.27 \%)$ & $7(17.95 \%)$ \\
\hline Others & $2307(11.30 \%)$ & $3(1.68 \%)$ & $1.30 \%$ & $0(0.00 \%)$ & $1(1.43 \%)$ & $0(0.00 \%)$ & $2(5.13 \%)$ \\
\hline Total & $20409(100.00 \%)$ & $179(100.00 \%)$ & $8.77 \%$ & $26(14.53 \%)$ & $\begin{array}{l}70 \\
(39.11 \%)\end{array}$ & $44(24.58 \%)$ & $39(21.79 \%)$ \\
\hline
\end{tabular}

\section{The correlations between maternal age and the incidence of fetal SCAs.}

Logistic regression analysis was performed to analyze the correlation between maternal age and the incidence of fetal SCAs, showed in Table 4. According to the results of logistic regression analysis, the incidence of all SCAs was significantly associated with maternal age $(P=0.002)$, and the odds ratio tended to increase by 1.044 times as maternal age increased by one year. The incidence of $45, X$ and $47, X X Y$ also showed significant correlation with maternal age $(P=0.017 ; P=0.000$, respectively), and the odds ratio of $45, \mathrm{X}$ tended to increase by 0.916 times and the odds ratio of $47, X X Y$ tended to increase by 1.127 times as maternal age increased by one year. However, the incidence of $47, X X X$ and $47, X Y Y$ was not found to be significantly correlated with maternal age $(P=0.473 ; P=0.272$, respectively). Trends in the incidence of fetal SCAs based on maternal age groups are shown in Fig. 3. The incidence of 47,XXY and all SCAs was basically positively correlated with maternal age, respectively, however, a significant inverse relationship with maternal age was found for $45, X$ at the upper end of the $29-33$ age range. Unlike the incidence of $47, X X X$ being completely independent of maternal age, the incidence of $47, X Y Y$ before the 34-38 years was completely dependent on the maternal age, and then decreased sharply.

Table 4

Regression coefficient and standard errors of logistic regression equations for fetal SCAs diagnosed in the study.

\begin{tabular}{|llllllll|}
\hline \multicolumn{7}{|c|}{ Constant term } & \multicolumn{7}{l|}{ Maternal term } \\
\hline & $\mathrm{A}$ & Standard error & $\mathrm{B}$ & Standard error & Odds ratio & $95 \% \mathrm{Cl}$ & $P$ value \\
\hline $45, \mathrm{X}$ & -3.861 & 1.143 & -0.088 & 0.037 & 0.916 & $0.852-0.984$ & $0.017<0.05$ \\
\hline $47, \mathrm{XXY}$ & -9.817 & 0.894 & 0.119 & 0.024 & 1.127 & $1.074-1.182$ & $0.000<0.05$ \\
\hline $47, \mathrm{XXX}$ & -6.807 & 0.953 & 0.020 & 0.028 & 1.020 & $0.966-1.078$ & $0.473>0.05$ \\
\hline $47, \mathrm{XYY}$ & -7.360 & 1.030 & 0.033 & 0.030 & 1.033 & $0.975-1.096$ & $0.272>0.05$ \\
\hline All SCA & -6.186 & 0.490 & 0.043 & 0.014 & 1.044 & $1.016-1.073$ & $0.002<0.05$ \\
\hline
\end{tabular}

\section{Comparison of the incidence of SCAs among different age groups.}


A chi-square test was performed to compare the incidence of fetal SCAs among different age groups; the statistical results are shown in Table 5. The incidence of all SCAs was significantly different among groups $\left(\chi^{2}=10.197, P=0.037\right)$, but there were no significant differences between adjacent groups $(P>0.05)$. The incidence of $45, X$ and $47, X X Y$ was also significantly different among groups $\left(\chi^{2}=10.977, P=0.027 ; \chi^{2}=29.159\right.$, $P=0.000$, respectively), while the incidence of $47, \mathrm{XXX}$ and $47, \mathrm{XYY}$ was not found to be significantly different among groups $\left(\chi^{2}=2.027, P=\right.$ $0.731 ; \chi^{2}=5.145, P=0.273$, respectively). We further compared the incidence of $45, X$ and $47, X X Y$ between adjacent groups, and found that the incidence of 45,X and 47,XXY was significantly different only between the 29-33 years and the 34-38 years $(P=0.004 ; P=0.002$, respectively) (Table 6). However, the incidence of $45, X$ in the $34-38$ years $(0.44 \%, 1: 2272)$ was lower than the $29-33$ years (2.35\%o, $1: 426)$, while the incidence of $47, \mathrm{XXY}$ was the opposite $(4.87 \%$, $1: 205$ vers $1.57 \%$, $1: 637)$.

Table 5

Comparison of the incidence of fetal SCAs among different age groups.

\begin{tabular}{|c|c|c|c|c|c|c|}
\hline Age groups & No. of patients & $45, X^{a}$ & $47, X X Y^{b}$ & $47, X X X^{c}$ & $47, X Y Y^{d}$ & All $\operatorname{SCA}_{s}{ }^{e}$ \\
\hline$\leq 23$ & 658 & $1(1.52)$ & $1(1.52)$ & $2(3.04)$ & $0(0)$ & $4(6.08)$ \\
\hline $24-28$ & 4304 & $8(1.86)$ & $4(0.93)$ & $8(1.86)$ & $5(1.16)$ & $25(5.81)$ \\
\hline $29-33$ & 5097 & $12(2.35)$ & $8(1.57)$ & $8(1.57)$ & $11(2.16)$ & $39(7.65)$ \\
\hline $34-38$ & 6780 & $3(0.44)$ & $33(4.87)$ & $18(2.65)$ & $18(2.65)$ & $72(10.62)$ \\
\hline $39+$ & 3570 & $2(0.56)$ & $24(6.72)$ & $8(2.24)$ & $5(1.40)$ & 39(10.92) \\
\hline Total & 20409 & $26(1.27)$ & $70(3.43)$ & $44(2.16)$ & $39(1.91)$ & 179(8.77) \\
\hline \multicolumn{7}{|c|}{ Data are presented as n (per 1000), unless otherwise indicated. } \\
\hline \multicolumn{7}{|c|}{ a The incidence of $45, X$ was significantly different among the groups $\left(\chi^{2}=10.977, P=0.027<0.05\right)$. } \\
\hline \multicolumn{7}{|c|}{ b The incidence of $47, X X Y$ was significantly different among the groups $\left(X^{2}=29.159, P=0.000<0.05\right)$. } \\
\hline \multicolumn{7}{|c|}{ c The incidence of $47, X X X$ was significantly different among the groups $\left(X^{2}=2.027, P=0.731>0.05\right)$. } \\
\hline \multicolumn{7}{|c|}{$d$ The incidence of $47, X Y Y$ was significantly different among the groups $\left(\chi^{2}=5.145, P=0.273>0.05\right)$. } \\
\hline \multicolumn{7}{|c|}{ e The incidence of all SCAs was significantly different among the groups $\left(\chi^{2}=10.197, P=0.037<0.05\right)$} \\
\hline
\end{tabular}

Table 6

Comparison of the incidence of $45, \mathrm{X}$ and $47, \mathrm{XXY}$ between the $29-33$ years and the $34-38$ years.

\begin{tabular}{|c|c|c|c|c|c|c|c|c|c|}
\hline \multirow[t]{2}{*}{ Age groups } & \multirow[t]{2}{*}{ No.of patients } & \multicolumn{4}{|l|}{$45, X$} & \multicolumn{4}{|l|}{$47, X X Y$} \\
\hline & & Number/Incidence & PPV & $x^{2}$ & $\mathrm{P}$ & Number/Incidence & PPV & $x^{2}$ & $\mathrm{P}$ \\
\hline $29-33$ & 5097 & $12(2.35)$ & $1: 426$ & \multirow[t]{2}{*}{8.432} & \multirow[t]{2}{*}{0.004} & $8(1.57)$ & $1: 637$ & \multirow[t]{2}{*}{9.198} & \multirow[t]{2}{*}{0.002} \\
\hline $34-38$ & 6780 & $3(0.44)$ & $1: 2272$ & & & $33(4.87)$ & $1: 205$ & & \\
\hline \multicolumn{10}{|c|}{ Data are presented as $n$ (per 1000), unless otherwise indicated. } \\
\hline \multicolumn{10}{|c|}{ PPV: positive predictive value } \\
\hline
\end{tabular}

\section{Discussion}

The present study reported one the largest cohorts of invasive prenatal diagnosis by amniotic fluid specimens from a representative database of a single cytogenetics laboratory in China. An advantage of the study was that the data was collected from the same prenatal diagnosis center, thus avoiding bias in the medical performance, and it was from a teaching hospital of well-known universities with a case ascertainment of approximately $100 \%$ with high data quality. In addition, the level of misdiagnosis could be determined by strictly following the national guideline and follow-up system. In the study, the incidence of all SCAs (8.77\%o, about 1/114), 45,X (1.27\%o, 1/787), 47,XXY (3.42\%o, 1/292), 47,XXX (2.16\%o, 1/463), 47,XYY (1.91\%o, 1/524) was much higher than earlier publication [4-5]. There were several factors that might affect this result. The most important reason might be that the current knowledge about the incidence of chromosomal abnormalities in the general population came from studies in newborns carried out in the 1970s, this data has not been updated and the earlier studies had technical and methodological limitations. Especially, amniocentesis was only performed on women who were already marked as higher risk. This is a different incidence rate than the earlier referenced incidence rate that was reported in terms of all live births. Second, as the largest prenatal diagnosis center and the main referral centers in Zhejiang Province, many patients with the referral indications of amniocentesis were likely to be referred 
to the hospital for further evaluation and management. And this study did not exclude pregnant women with family history of chromosomal abnormalities, ultrasound abnormalities, adverse pregnancy history and other indicators, which might increase the proportion of chromosomal abnormalities. Moreover, new screening technologies as alternatives had been used to detect chromosomal abnormalities, especially, the increasing reliability of NIPT has facilitated far more first trimester screening for the major aneuploidies [14]. NIPT as a successful application in routine clinical practice has been widely used to detect trisomy $21,18,13$ and SCAs [15]. In the present study, it was found that $19.00 \%$ of pregnant women with fetal SCAs had the indication of abnormal NIPT results, and the indication had the highest positive predictive value for SCAs (83.74\%o). However, it must be noted that NIPT has only been available in China since 2012, and given the length of the study period, this might have a significant impact on the result. The discrepancy might also be related to the age distribution of the participants since the frequency of some chromosomal abnormalities was directly associated with maternal age [16]. In this study, the percentage of AMA women has gradually increased since 2012, especially in 2016 and 2017 reaching 56.04\% and 57.57\%, respectively, which might be due to the implementation of the "second-child policy" in China. In addition, due to the incompleteness of the data, this study failed to rule out confounding factors such as maternal parity history and father's age, however, previous studies have found that fetal chromosomal aneuploidies might be related to these factors [17-18]. Therefore, it was reasonable to have a higher incidence rate of fetus SCAs.

The incidence of fetal SCAs depends on clinical indications [19]. AMA was the most frequent referral indication for 47, $X X Y, 47, X X X$ and $47, X Y Y$, while abnormal ultrasound findings was the most frequent referral indication for $45, X$, which was consistent with the previous study [20]. This study indicated that the incidence of fetal SCAs was significantly related to maternal age, ranging from the lowest of 5.81 per 1,000 fetuses at the 24-28 years to the highest of 10.92 per 1,000 fetuses at the $39+$ years. The incidence of fetal SCAs was significantly different among groups, but not significantly different between adjacent groups. This study also found that the incidence of $45, X$ and $47, X X Y$ was significantly related to maternal age, while the incidence of $47, X X X$ and $47, X Y Y$ was not related to maternal age. And the incidence of $45, X$ and $47, X X Y$ was significantly different only between the 29-33 years and the 34-38 years. Specifically, unlike the incidence of 47, XXY, which gradually increased with advancing maternal age, the incidence of $45, X$ reached the peak of $2.35 \%$ o $(1: 426)$ at the $29-33$ years, and then decreased sharply to $0.44 \%$ o (1: 2272) at the 34-38 years. However, because the number of research subjects for each type of abnormality was too small, we could not tell at what age the cutoff occured.

Turner's syndrome is the only complete monosomy that is viable in human beings. Some studies have shown that the loss of the $\mathrm{X}$ chromosome in the peripheral blood lymphocytes increased with maternal age [21-23], but clinical studies have shown that young women have a higher incidence of $45, X$ [24]. However, the incidence of $45, X$ increased with maternal age and reached the peak at a specific maternal age, then tended to decline at the upper limit of the age range, which was consistent with as Ferguson-Smith et al described [9]. Does this mean that when the age of pregnant women reaches a certain threshold, the incidence of $45, X$ will decline? Does this happen by accident? Uematsu $A$ [25] found that $45, X$ is not related to advanced maternal age and it is more likely due to instability of the $Y$ chromosome since $75-80 \%$ of $X$ chromosomes in patients with $45, X$ are maternal in origin. The study failed to rule out confounding factors such as father's age, which required expanding the sample size to seek possible influencing factors in the further studies.

$47, \mathrm{XXY}$ is the most frequent genetic disorder and accounts for approximately two-thirds of all the cytogenetic abnormalities [26]. In the study, $47, X X Y$ was the most common SCAs and accounted for $39.11 \%$ of all SCAs. Similar to previous studies [9-11], we found that $47, X X Y$ showed significant correlation with maternal age. The incidence of $47, X X Y$ was basically positively correlated with maternal age, except for the $\leq 23$ years, which might be due to the number of patients and affected fetuses was very small in size. According to the results of this study, the incidences of $47, X X X$ and $47, X Y Y$ were not found to be significantly correlated with maternal age. However, trend in the incidence of fetal SCAs showed that the incidence of $47, X X X$ was completely independent of maternal age, while the incidence of $47, X Y Y$ was completely dependent on the maternal age before the 34-38 years and then decreased sharply at the 39+years, this necessitates further studies on larger cohorts in the future. In addition, the incidence of $47, X X Y, 47, X X X$ and $47, X Y Y$ was found to increase dramatically at the age of 44 . However, because the number of cases at the age of 44 or older was very small, analysis of the correlation between fetal chromosomal abnormalities and extreme maternal age was not possible.

\section{Conclusion}

Maternal age was ascertained to be a strong risk factor for fetal SCAs, and the incidence of fetal SCAs depended on clinical indications. This study compared the incidence of common fetal SCAs in pregnant women of different ages throughout the child-bearing period that not only limited to advanced age women, which could better reflect the relationship between fetal SCAs and maternal age.

\section{Materials And Methods}

Subjects 
This was a retrospective cohort study of all consecutive singleton pregnancies who underwent second-trimester amniocentesis between January 1st 2011 and December 31st 2018 at the Women's Hospital of Zhejiang University. Amniocentesis and karyotype analysis was performed in 20,672 pregnant women during the last 8 years. Among these, 253 cases were excluded for multiple gestations and 10 cases were excluded for failing in amniotic fluid culture. Eventually, 20,409 singleton amniotic fluid specimens were eligible for the study. All pregnant women underwent amniocentesis at the $19-22^{+6}$ weeks of gestation. Gestational week was determined based on the date of the last menstrual period (LMP) or ultrasonic examination (biparietal diameter) if the value differed from the LMP-derived gestational week by $\geq 3$ days. The indications for amniocentesis have strictly followed the guideline of Chinese government, including advanced maternal age (AMA) (35 years or older at the expected date of delivery), positive results of maternal serological screening (MSS) (T21 $\geq 1 / 270, T 18 \geq 1 / 350)$, abnormal ultrasound findings (including increased NT before the first-trimester screening, fetal structural abnormalities, abnormal ultrasound soft markers), positive results from noninvasive prenatal testing (NIPT), family history of chromosomal abnormalities (a previous child with chromosomal abnormalities or paternal/maternal carrying chromosomal abnormalities, including sex chromosomal abnormalities), adverse pregnancy history (a history of intrauterine fetal death or aborted fetuses), parental anxiety and others. In the study, maternal age was used as the only indication for statistical analysis, regardless of the main referral indications for amniocentesis. Traditionally, prenatal diagnosis has been offered to women aged 35 years or older at the expected date of delivery. This study was based on maternal age at the time of amniocentesis, not the expected date of delivery. Most of 34-year-old pregnant women would give birth at the age of 35-year old, so we classified the 34-year-old women to the advanced age group. The cases were categorized into the following 5 age groups at intervals of 5 years according to maternal age ( $\leq 23,24-28,29-33,34-$ $38,39^{+}$years) and entered as a categorical variable in all samples. The patients in only 270 cases were $\geq 44$ years of age; they were included in the 39-43-year-old group (39+ years). In this study, we calculated the incidence of common fetal SCAs (45,X; 47,XXX; 47,XXY; 47,XYY), eXcluding rare fetal SCAs such as 48,XXXX,48,XXXY, 48,XXYY and 69,XXX. This study was approved by the Scientific Research Ethics Committee of the Women's Hospital of Zhejiang University. This was a retrospective study of the clinical database with no intervention and no informed consent was required.

\section{Karyotyping}

Conventional cytogenetic analysis was performed on all samples according to the standard protocol of the Women's Hospital of Zhejiang University of Human Cytogenetics Guidelines. Amniotic fluid was centrifuged and cultured immediately after the specimen was obtained. 20 mL amniotic fluid (with the first 1-2 mL amniotic fluid was discarded) was divided into two sterile centrifuge tubes for centrifugation at 1500 rpm for $10 \mathrm{~min}$, and the supernatant was discarded. The cell suspension was inoculated into culture bottle (Corning, Falcon ${ }^{\circ}$, catalog number: 353108 ) containing of $5 \mathrm{~mL}$ amniotic fluid cell medium (BIO-AMF-2., Biological Industries Ltd, Kibbutz Beit-Haemek, Israel) in $5 \%$ CO ${ }_{2}$ incubator at $37^{\circ} \mathrm{C}$ for 6-7 days, then the medium was changed. The cell growth was observed every day after the medium was changed. When the amniotic fluid cells adhered to the wall and exhibited multiple clones under an inverted microscope, the amniotic fluid cells in each culture bottle were collected separately. Metaphase chromosomes with targeted 400-band level were obtained by hypotension, fixation, trypsinization, Giemsa staining, and so forth. Cytogenetic analysis was performed by Leica GLS120 Automated Nuclear Scanning System (CytoVision., Leica, Wetzlar, German) after G-banding, and at least 30 metaphases were counted and 5 metaphases were analyzed for each patient. If there were different cell lineages in the same patient, increased the count to 50-100 metaphases to establish the mosaicism. Karyotype results were described by certified physicians following the criteria established by the International System for Human Cytogenetic Nomenclature guidelines (ISCN, 2016, 5th edition) [13]. Lymphocyte karyotype analysis of the couples was recommended in cases of chromosomal structural abnormalities.

\section{Statistical analysis}

The data used for analysis did not contain identifiable personal information to protect individuals' privacy. Data were presented as mean \pm SD and $\mathrm{n}(\%$ or \%o). Logistic regression analysis was performed to analyze the correlation between maternal age and fetal SCAs. Chi-square test was performed to compare the incidence of fetal SCAs among age groups. Fisher's exact test was used when there was a frequency count $<5$. $P$ $<0.05$ was considered statistically significant. Moreover, we calculated the odds ratio (OR) and confidence interval (CI) at $95 \% \mathrm{Cl}$. SPSS version 16.0 (SPSS Inc., Chicago, IL , USA) was used for the statistical analysis.

\section{Abbreviations}

SCAs: Sex chromosome aneuploidies; LMP: last menstrual period; AMA: advanced maternal age; NIPT: noninvasive prenatal testing; MSS: maternal serological screening; ISCN: International System for Human Cytogenetic Nomenclature guidelines; OR:odds ratio; Cl:confidence interval

\section{Declarations}




\section{Ethics approval and consent to participate}

This study was approved by the Scientific Research Ethics Committee of Women's Hospital, School of Medicine, Zhejiang University. This was a retrospective study of the clinical database with no intervention and no informed consent was required.

\section{Consent for publication}

The manuscript does not contain any individual person's data.

\section{Availability of data and materials}

The datasets generated during the current study are available from the corresponding author on a reasonable request.

\section{Competing interests}

The author declares that he has no competing interests.

\section{Funding}

None declared.

\section{Authors' contributions}

HGL is fully responsible for this editorial.

\section{Acknowledgments}

We should like to thank our patients for agreeing to donate their personal data and allowing the data to be published.

\section{Availability of data and materials}

The data supporting the conclusions of this article is included within the article.

\section{References}

1. Dey M, Sharma S, Aggarwal S. Prenatal Screening Methods for Aneuploidies. N Am J Med Sci. 2013,5(3),182-190.

2. Fauret AL, Bilan F, Patri S, Couet D, Marechaud M, Pierre F, et al. Molecular biology usefulness for rapid diagnosis of Down's syndrome and common aneuploidies. Gynecol Obstet Fertil. 2009;37(7-8): 611-619.

3. Thompson MW. Thompson and Thompson: Genetics in Medicine (5 $5^{\text {th }}$ edn). Toronto: WB Saunders, 1991.

4. Hong DS, Reiss AL. Cognitive and neurological aspects of sex chromosome aneuploidies. Lancet Neurol. 2014;13(3):306-318.

5. Skuse D, Printzlau F, Wolstencroft J. Sex chromosomeaneuploidies. Handb Clin Neurol.2018;147:355-376.

6. Li H, Li Y, Zhao R, Zhang Y. Cytogenetic Analysis of Amniotic Fluid Cells in 4206 Cases of High-Risk Pregnant Women. Iran J Public Health. 2019;48(1):126-131.

7. Hassold T, Hall H, Hunt P. The originof humananeuploidy: where we have been, where we are going. Hum Mol Genet. 2007;16:203-208.

8. Heffner LJ. Advanced maternal aged how old is too old? N Engl J Med 2004;351(19):1927-9.

9. Ferguson-Smith MA, Yates JR. Maternal age specific rates for chromosome aberrations and factors influencing them: report of a collaborative european study on 52965 amniocenteses. Prenat Diagn. 1984;4:5-44.

10. Zhu YN, Lu SM, Bian X, Bian XM, Wang H, ZhuBS, et al. A multicenter study of fetal chromosomal abnormalities in Chinese women of advanced maternalage. Taiwan J Obstet Gynecol. 2016;55(3):379-384. 
11. Forabosco A, Percesepe A, Santucci S. Incidence of non-age-dependent chromosomal abnormalities: a population-based study on 88965 amniocenteses. Eur J Hum Genet. 2009;17(7):897-903.

12. Kim YJ, Lee JE, Kim SH, Shim SS, Cha DH. Maternal age-specific rates of fetal chromosomal abnormalities in Korean pregnant women of advanced maternal age. Obstet Gynecol Sci. 2013;56(3):160-166.

13. Jean McGowan-Jodan, Aneet Simons, Michael Schmid. An International System for Human Cytogenetic Nomenclature. Switzerland: S. Kargerpress; 2016. p38.

14. Wapner R, Thom E, Simpson JL,Pergament E, Silver R, Filkins K, et al. First-trimester screening for trisomies 21 and 18 . N Engl J Med; 2003 ; 349(15): 1405-1413.

15. Goodwin S, McPherson JD, McCombie WR. Coming of age: ten years of next-generation sequencing technologies. Nat Rev Genet. 2016;17(6):333-351.

16. Driscoll DA, Gross S. Clinical practice. Prenatal screening for aneuploidy. N Engl J Med. 2009; 360(24):2556-2562

17. Carothers AD, Collyer S, De Mey R, Frackiewicz A. Parental age and birth order in the aetiology of some sex chromosome aneuploidies. Ann Hum Genet. 1978;41(3):277-287.

18. Erickson JD. Down syndrome, paternal age, maternal age and birth order. Ann Hum Genet. 1978;41(3):289-298.

19. Shaffer LG, Rosenfeld JA, Dabell MP, Coppinger J, Bandholz AM, Ellison JW, et al. Detection rates of clinically significant genomic alterations by microarray analysis for specificanomalies detected by ultrasound. Prenat Diagn. 2012;32(10):986-995.

20. Nishiyama M, Yan J, Yotsumoto J, Sawai H, Sekizawa A, Kamei Y, et al. Chromosome abnormalities diagnosed in utero: a Japanese study of 28983 amniotic fluidspecimens collected before 22 weeks gestations. J Hum Genet. 2015; 60(3):133-137.

21. Fitzgerald PH. A mechanism of X chromosome aneuploidy in lymphocytes of aging women. Humangenetik. 1975,28(2):153-158.

22. Fitzgerald $\mathrm{PH}, \mathrm{McEwan} \mathrm{CM}$. Total aneuploidy and age-related sex chromosome aneuploidy in cultured lymphocytes of normalmen and women. Hum Genet. 1977;39(3):329-337.

23. Jarvik LF, Yen FS, Fu TK, Matsuyama SS. Chromosomes in old age: a six year longitudinal study. Hum Genet. 1976;33(1):17-22.

24. Heffner LJ. Advanced maternal age-how old is too old? N Engl J Med. 2004 ;351(19):1927-1929.

25. Uematsu A, Yorifuji T, Muroi J, Kawai M, Mamada M, Kaji M, et al. Parental origin of normal X chromosomes in Turner syndrome patients with various karyotypes: implications for the mechanism leading to generation of a 45,X karyotype. Am J Med Genet. 2002;111(2):134-139.

26. De-Braekeleer M, Dao TN. Cytogenetic studies in male infertility: a review. Hum Reprod. 1991;6(2):245-250.

\section{Figures}

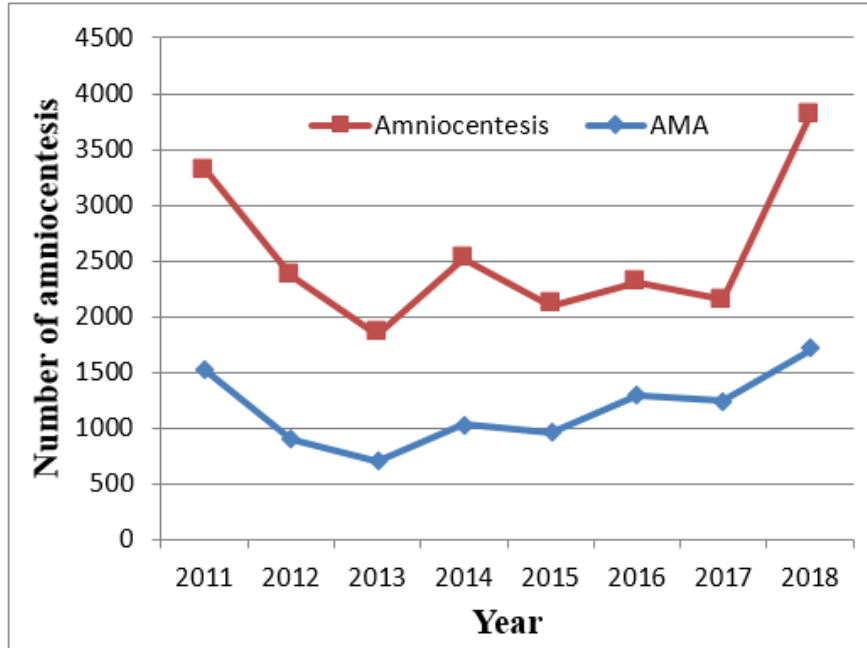

Fig 1.(a)

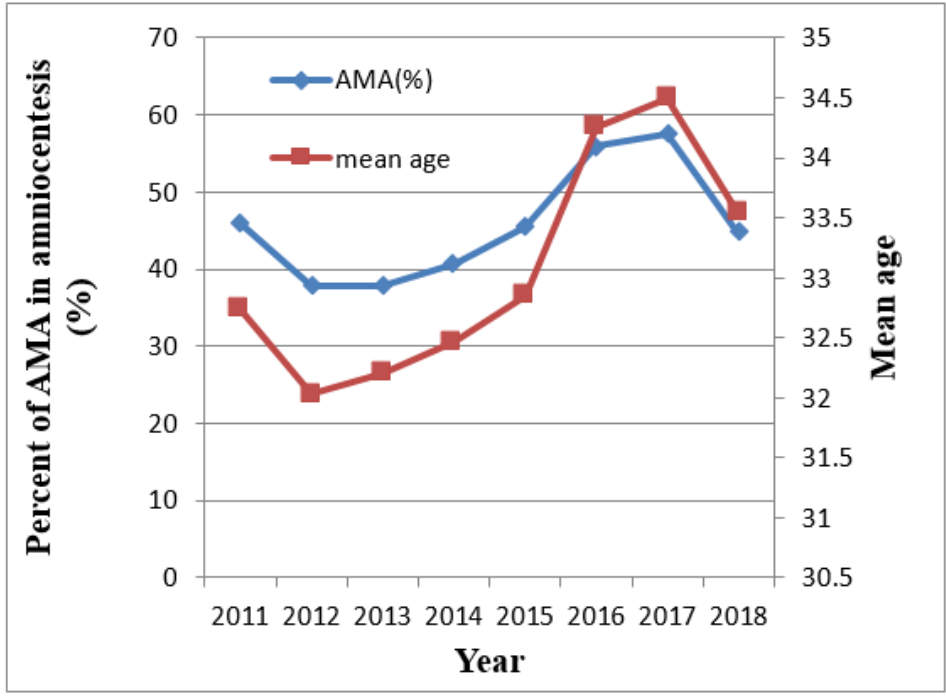

Fig 1.(b)

\section{Figure 1}

(a). Change in the relative number of amniocentesis and women with advanced maternal age ( $\geq 35)$ undergoing amniocentesis at the Women's Hospital of Zhejiang University, 2011-2018. (b). Trend in the proportion of women with advanced maternal age undergoing amniocentesis at the 
Women's Hospital of Zhejiang University, and mean age of these women, 2011-2018.

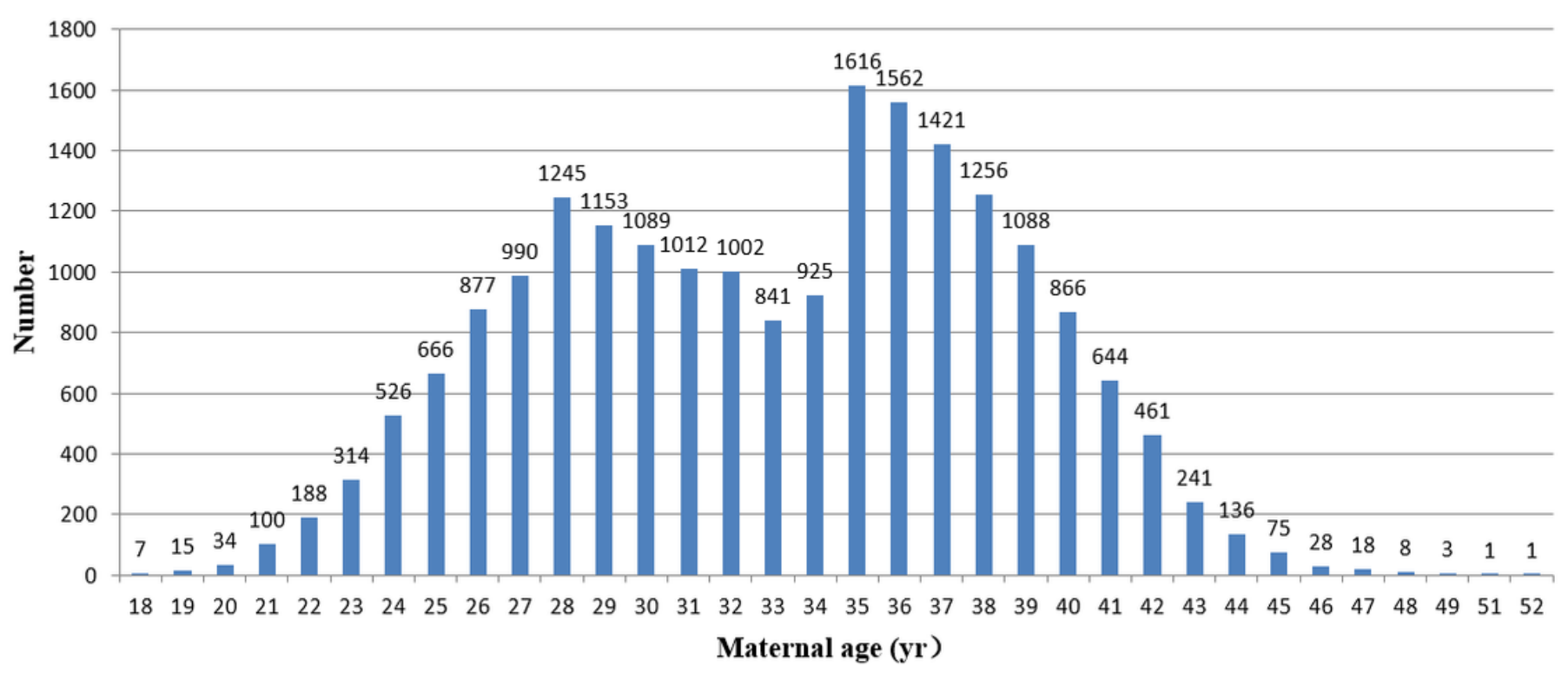

\section{Figure 2}

Maternal age distribution in the entire study cohort. 

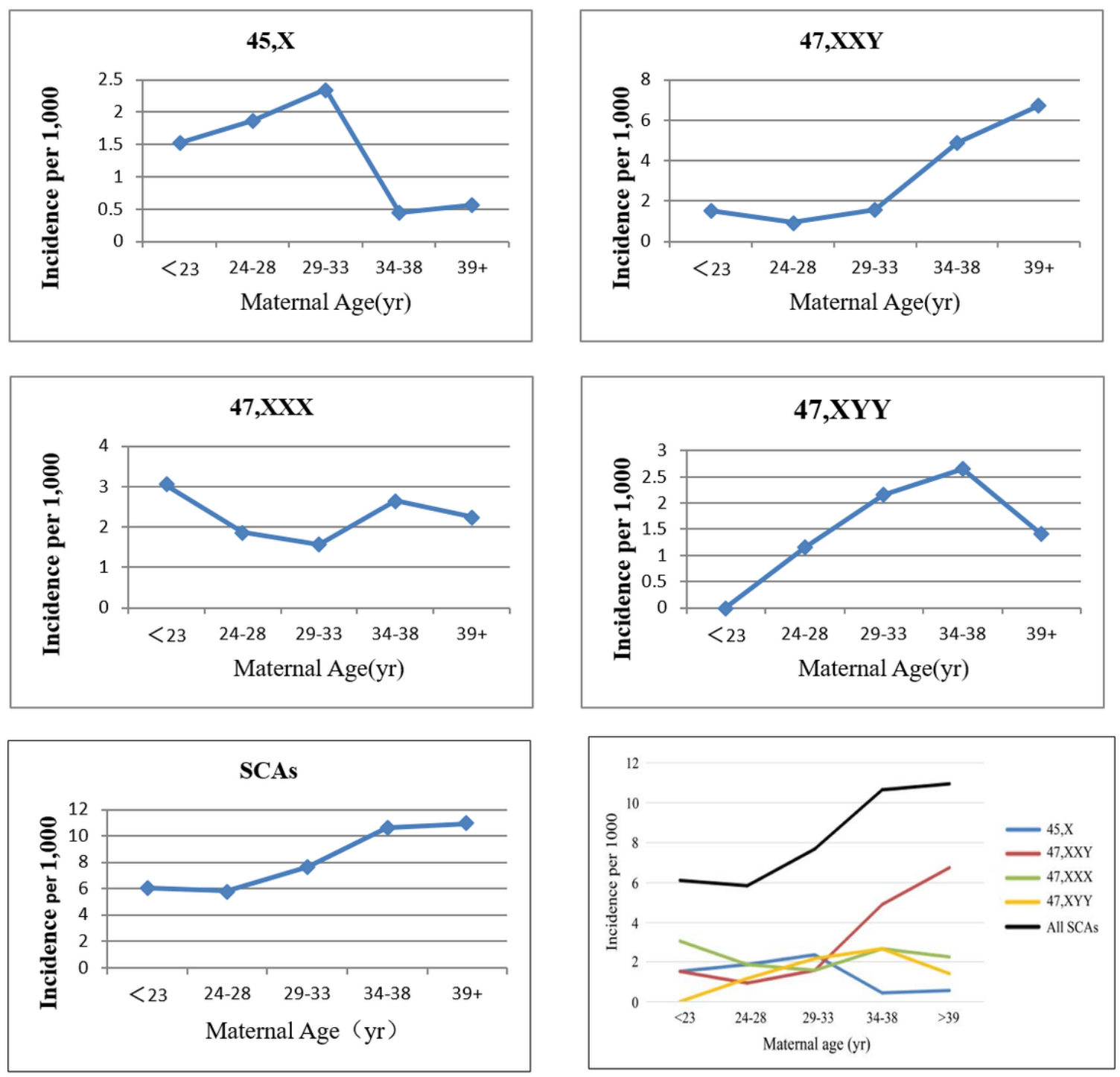

\section{Figure 3}

Trend in the incidence (per 1000) of fetal SCAs based on maternal age groups. 